\title{
Editorial: Advances in Pediatric Pulmonology
}

\author{
Andrew Bush ${ }^{1} \cdot$ Sushil K. Kabra ${ }^{2}$ \\ Received: 19 May 2015 / Accepted: 19 May 2015 / Published online: 19 June 2015 \\ (C) Dr. K C Chaudhuri Foundation 2015
}

Respiratory illnesses are major causes of morbidity and mortality in children. They may involve any part of respiratory system including upper airways, lower airways, and the gastrointestinal, cardiovascular, musculoskeletal and neuromuscular systems. Hence it is particularly appropriate that the IJP has commissioned a series on respiratory diseases and their management in children, and it has been an honour for us to co-edit it.

Objective documentation of pulmonary physiology should play an important role in diagnosis and monitoring of most respiratory illnesses. Various methods of testing pulmonary physiology in adults are well established. Some of these methods have been adopted for children also. However, most methods need active cooperation which is difficult to obtain in children below $7 \mathrm{y}$ of age. In addition, it is often difficult to interpret the results. Over the past decades, some new tests that require only passive cooperation have been developed, together with data enabling proper interpretation.

In this issue of IJP, Sonnappa has reviewed various methods of pulmonary function testing in children. She describes methods to be used for assessment of airway resistance in various obstructive airway diseases, measurement of lung volumes, measurement of gas exchange and exercise testing. The article gives valuable guidance on which test to use and how to interpret the results [1].

Sushil K. Kabra

skkabra@hotmail.com

1 Department of Pediatrics, Imperial College, London, UK; Department of Pediatric Respiratory Medicine, National Heart and Lung Institute, London, UK and Department of Pediatric Respiratory Medicine, Royal Brompton \& Harefield NHS Foundation Trust, London, UK

2 Division of Pediatric Pulmonology, Department of Pediatrics, All India Institute of Medical Sciences, New Delhi 110029, India
Cystic fibrosis (CF) is a very common inherited life limiting disease. It was previously considered to be disease of Caucasians only. However, more recently it has been reported from all over world; and it is clear that in many developing world settings, where so many previously healthy infants die of diarrhea and pneumonia, the diagnosis has been frequently missed. Significant progress has been made in the management of CF over the past 5-6 decades. After identification of the genetic mutation of CF in 1989, there was great hope of a cure for $\mathrm{CF}$. Treatment paradigms have changed from fighting the downstream consequences of the mutation (e.g., infection and inflammation) to specific molecular therapies; gene repair, gene therapy and mutation class specific designer medications. An orally active medication, Ivacaftor has been approved, initially for use in patients with the G551D mutation, and latterly for the other Class III gating mutations. There are now several reports on mutation specific treatment for CF [2]. In this issue of IJP, Guglani has reviewed all ongoing studies on advances in treatment of CF [3]. The article will be helpful in understanding the advances in $\mathrm{CF}$ and will be a step towards improvement in the management of these children.

Upper airway obstruction is a common and frightening cause of acute as well as chronic illness in children. Some of these illnesses present as an acute emergency and need immediate intervention, while other conditions are chronic and mandate long term management. In this issue Mandal et al. [4] describe various causes of airway obstruction, their diagnosis and treatment, which should assist all dealing with these conditions.

Acute lower respiratory tract infection or pneumonia is the leading cause of mortality in under five year old children. There has been a significant improvement in the survival of children with pneumonia after the introduction of an acute respiratory tract infection (ARI) control program about three decades ago. Changes have been done in ARI program including change in first line antibiotics. In this issue, Qin and Shen [5] have reviewed the management of community acquired pneumonia, to provide a guideline for the clinicians. 
We believe these four articles in this issue of IJP, written by experts from different parts of the world, will improve our understanding of respiratory illnesses in children, and will help in improving the care of children in a village, that is the world.

Conflict of Interest None.

Source of Funding None.

\section{References}

1. Sonnappa S. The tools of the trade - physiological measurements of the lungs. Indian J Pediatr. 2015. doi:10.1007/s12098-015-1787-2.

2. Hoffman LR, Ramsey BW. Cystic fibrosis therapeutics: the road ahead. Chest. 2013;143:207-13.

3. Guglani L. Changing the paradigm - treating the basic defect in cystic fibrosis. Indian J Pediatr. 2015. doi:10.1007/s12098-015-1786-3.

4. Mandal A, Kabra SK, Lodha R. Upper airway obstruction in children. Indian J Pediatr. 2015. doi:10.1007/s12098-015-1811-6.

5. Quin Q, Shen KL. Community-acquired pneumonia and its complications. Indian J Pediatr. 2015. doi:10.1007/s12098-015-1785-4. 